\title{
Testing Hypothesis for New Class of Life Distribution NBUFR-t0
}

\author{
N. A. Abdul Alim \\ Mathematics Department, Faculty of Science Al-Azhar University \\ Nasr city, 11884, Cairo, EGYPT
}

\begin{abstract}
A new concept of ageing distribution, namely new better (worse) than used in failure rate at specific time $t_{0}$ (NBUFR- $t_{0}$ (NWUFR- $\left.t_{0}\right)$ ) are introduced. The problem is investigated how to prove that after a specified time $t_{0}$ of operation the failure rate of an item is greater than the corresponding failure rate of a new item. This problem occurs in various areas like for instance in industry, when designing a maintenance policy. A test statistics that based on the goodness of fit method are derived for testing exponentially versus the NBUFR- $t_{0}$ alternatives. The percentiles and powers of this test statistic are tabulated. The asymptotic efficiencies for some alternatives are derived. A medical data is taken as a practical application.
\end{abstract}

\section{Keywords}

NBUFR- $t_{0}$; NWUFR- $t_{0}$; Mont Carlo method; Hypotheses testing; NBUFR; U-statistic; Life testing; Exponential distribution; Goodness of fit testing; Efficiency; Power of test.

\section{INTRODUCTION}

The notion of aging for engineering systems has been characterized by several classes of life distributions in reliability. Various classes of life distributions have been introduced to describe several types of deterioration (improvement) that accompany aging. Unfortunately these types of classes are restricted to one type of applications since they present positive aging or negative aging for their dual classes throughout their life span of the underlying components or their systems.

[16] have introduced the new better than used at specific age at $t_{0}$ (NBU- $t_{0}$ ) and its dual (NWU- $t_{0}$ ). They have listed some types of situation where (NBU- $\left.t_{0}\right)$ aging or its dual might arise. [12] introduced the new better than used in convex ordering at age $t_{0}$ (NBUC- $t_{0}$ ), and its dual.

There are many situation in real life where the components of the system gradually deteriorate up time $t_{0}$ which is warranty guarantee time provided by most manufacturers, then maintenance through repairs or spare parts replacement take place after time $t_{0}$. Here maintenance is expected to improve the performance of the system but can not bring it back to a better situation than it was at age $t_{0}$. For some interesting examples, see [16].

One of the oldest problems in aging distributions is testing exponentiality against the known classes of aging distributions such as IFR, IFRA, NBU, NBARFR and others, for example see [23], [15], [2], [3] and [20] among others. With respect to the NBUFR class, see [6].

[16] introduced the concept of the class of life distributions called new better than used of specified age $t_{0}$. [11], [22] and [4] are investigated the testing of new better than used of specified age $t_{0}$ (NBU- $\left.t_{0}\right)$ alternatives and others as [19] deal with the problem with respect to increasing failure rate (IFR) and new better than used (NBU) after specified time $t_{0}$. [21] introduce the new better (worse) than used renewal failure rate at specied time $t_{0}$ NBURFR- $t_{0}$ (NWURFR- $t_{0}$ ).

In practice, one might be interested in the new better than used failure rate behaviour at specified age $t_{0}$. Therefore a new concept of ageing distribution namely new better (worth) than used failure rate of specified age $t_{0}$ (NBUFR$t_{0}$ (NWUFR- $\left.\left.t_{0}\right)\right)$. This class is generalization of NBUFR ([1]). We investigate the testing exponentiality problem of it alternatives here according to goodness of fit approach. The distributional properties of the test statistic are studied. The performances of a proposed test are studied in terms of powers for some of alternative life distributions. The proposed test is shown to be consistent for NBUFR- $t_{0}$ alternatives.

This paper is organized as follows. In Section 2 we will recall some definitions and facts about characteristics of lifetime distributions and about relationship between some classes of life distributions. Section 3 introduced main results for our study represented in the test statistic proposed for testing exponentially versus the NBUFR- $t_{0}$ alternatives.

\section{DEFINITIONS and RELATIONSHIPS}

Let $\mathrm{X}$ be a non-negative random variable representing the lifetime of a device, with distribution function $F(x)=P[X \leq x], \quad$ survival function $\bar{F}(x)=1-F(x)$ and density function $\mathrm{f}(\mathrm{x})$ (if it exists), then the corresponding failure rate $r(t)$, when the distribution is absolutely continuous, is defined by

$$
r(t)=\lim _{\delta t \rightarrow 0} \frac{P[T \leq t+\delta t \mid T \geq t]}{\delta t} .
$$

[1] introduced the new better than used failure rate class of life distribution and its dual and discussed some of its properties as follows

Definition 2.1 An absolutely continuous probability distribution $\mathrm{F}$ on $[0, \infty)$ for which $\mathrm{F}(\mathrm{x}) / \mathrm{x}$ has a limit as $\mathrm{x} \rightarrow 0$ from above is said to be NBUFR(NWUFR) if there exists a version $r$ of the failure function such that

$$
\tilde{r}(0) \leq(\geq) r(t)
$$


Where

$$
\tilde{r}(0)=\lim _{t \rightarrow 0^{+}} t^{-1} \int_{0}^{t} r(x) d x .
$$

Authors state that: An absolutely continuous distribution $\mathrm{F}$ on $[0, \infty)$ for which $\mathrm{F}(\mathrm{x}) / \mathrm{x}$ has a limit as $\mathrm{x} \rightarrow 0$ from above has the NBUFR property iff

$$
\bar{F}(x+t) \leq e^{-\tilde{r}(0) t} \bar{F}(x), \text { for all } \mathrm{x}, \mathrm{t} \geq 0
$$

[7] introduced the new better than used (NBU) and its dual new worse than used (NWU) classes of life distributions as follows

Definition 2.2 The random variable $X$, or its distribution function $\mathrm{F}(\mathrm{x})$, is said to be (or to have) new better (worse) than used (NBU (NWU)) if

$$
\bar{F}(x+t) \leq(\geq) \bar{F}(x) \bar{F}(t), \text { for all } \mathrm{x}, \mathrm{t} \geq 0
$$

[9] introduced the class of new better than used in convex ordering (NBUC) distributions. [10] suggested the class of new better than used of second order(2) as follows.

Definition 2.3 The random variable $\mathrm{X}$, or its distribution function $\mathrm{F}(\mathrm{x})$, is said to be

new better than used in convex order (NBUC) if

$$
\int_{x}^{\infty} \bar{F}(t+s) d s \leq \bar{F}(t) \int_{x}^{\infty} \bar{F}(s) d s, \text { for all } \mathrm{x}, \mathrm{t} \geq 0
$$

new better than used in concave order (NBU(2)) if

$$
\int_{0}^{x} \bar{F}(t+s) d s \leq \bar{F}(t) \int_{0}^{x} \bar{F}(s) d s, \text { for all } \mathrm{x}, \mathrm{t} \geq 0
$$

We have the following chain of implications of the above mentioned classes as follows:

and

$$
\mathrm{NBU}(2) \Leftarrow \mathrm{NBU} \Leftarrow \mathrm{NBUC}
$$

$$
\text { NBU } \Rightarrow \text { NUBE } \Rightarrow \text { NBUFR }
$$

[16] introduced the larger class of life distributions called new better than used of age $t_{0}$ defined as follows:

Definition 2.4 A non-negative random variable $X$ is said to be new better than used of age $t_{0}$ if

$$
\bar{F}\left(t_{0}+x\right) \leq \bar{F}\left(t_{0}\right) \bar{F}(x) \text {, for all } \mathrm{x} \geq 0
$$

The NBU property states that a used item of any age has stochastically smaller residual life length than does a new item, whereas the NBU- $t_{0}$ property states that a used item of age $t_{0}$ has stochastically smaller residual life length than does a new item.

[12] introduced the concept of new better than used in convex ordering and new better than used of second order (2) classes of age $t_{0}$ as follows.

Definition 2.5 A non-negative random variable $\mathrm{X}$ is said to be new better than used in convex ordering of age $t_{0}$ (NBUC$\left.t_{0}\right)$ if

$$
\int_{x}^{\infty} \bar{F}\left(t_{0}+y\right) d y \leq \bar{F}\left(t_{0}\right) \int_{x}^{\infty} \bar{F}(y) d y, \text { for all } \mathrm{x} \geq 0
$$

new better than used of second order(2)of age $t_{0}$ (NBU(2)$\left.t_{0}\right)$ if

$$
\int_{0}^{x} \bar{F}\left(t_{0}+y\right) d y \leq \bar{F}\left(t_{0}\right) \int_{0}^{x} \bar{F}(y) d y, \text { for all } \mathrm{x} \geq 0
$$

It is seen from the above definitions that that

$$
N B U C-t_{0} \Leftarrow N B U-t_{0} \Rightarrow N B U(2)-t_{0}
$$

and

$$
\begin{gathered}
N B U-t_{0} \\
\Downarrow
\end{gathered}
$$

$$
N B U C-t_{0} \Rightarrow N B U E-t_{0} \Leftarrow N B U(2)-t_{0} .
$$

By the same concept, we can introduce new class of life distributions at specific age $t_{0}$. Namely new better than used in failure rate at specific age $t_{0}$ (NBUFR- $t_{0}$ (NWUFR- $\left.t_{0}\right)$ ) as follows.

Dfinitione 2.6 An absolutely continuous distribution F on [ $\left.t_{0}, \infty\right)$ is said to be new better (worse) than used in failure rate at specific time $t_{0}$ (NBUFR- $t_{0}$ (NWUFR- $\left.t_{0}\right)$ ) if there exist a version $r$ of the failure rate functions such that for specified $t_{0}$

$$
r\left(t_{0}\right) \leq(\geq) r\left(t_{0}+t\right), \text { for all } \mathrm{t} \geq 0
$$

Note that the border class where $r\left(t_{0}\right)=r\left(t+t_{0}\right)$ include only the following members:

all exponential distribution.

all life distribution defined on $\left[0, t_{0}\right]$.

all life distribution defined freely on $\left[0, t_{0}\right]$ and defined as $\bar{F}^{j}\left(t_{0}\right) \bar{F}\left(x-j t_{0}\right)$ for all $j t_{0} \leq x \leq(j+1) t_{0}, j \geq 1$.

\section{The U-STATISTIC TEST PROCEDURE}

In this section we consider the problem of testing $H_{0}: \bar{F}$ belongs to border distributions against $H_{1}: \bar{F}$ is strictly NBUFR- $t_{0}$ by using a goodness of fit approach. For more detail see [3]. The following is the form of our new class that called new better than used failure rate of specified age $t_{0}$

$r_{F}\left(t_{0}\right) \leq r_{F}\left(t+t_{0}\right), t \geq 0$ for fixed $t_{0}$,

that is equivalent to

$$
\frac{f\left(t_{0}\right)}{\bar{F}\left(t_{0}\right)} \leq \frac{f\left(t+t_{0}\right)}{\bar{F}\left(t+t_{0}\right)}, t \geq 0 \text { and fixed } t_{0} .
$$

In contrast to goodness of fit problems, where the tes statistic is based on a measure of departure from $H_{0}$ and $H_{1}$ , we refer to the departure between the two sides of inequality by

$$
\left.\Delta_{F}=\int_{0}^{a}\left(\bar{F}\left(t_{0}\right) f\left(x+t_{0}\right)-f\left(t_{0}\right) \bar{F}\left(x+t_{0}\right)\right)\right) d F_{0}(x)
$$

A nonparametric test statistic for this class is introduced. This test is designed to test the hypothesis whether a 
NBUFR- $t_{0}$ elements are strictly or not. The following lemma is essential for the development of our test statistic.

Lemma Let $X$ be a random variable with NBUFR- $t_{0}$ distribution function $\mathrm{F}$ then

$\Delta_{F}=$

$\bar{F}\left(t_{0}\right) E\left[e^{-\left(X-t_{0}\right)} I\left(X>t_{0}\right)\right]-f\left(t_{0}\right) E\left[1-e^{-\left(X-t_{0}\right)} I\left(X>t_{0}\right)\right]$

Proof Since

$$
\begin{aligned}
& \int_{0}^{\infty} \bar{F}\left(t+t_{0}\right) d F_{0}(t)=\int_{0}^{\infty} I\left(X>x+t_{0}\right) e^{-x} d x \\
= & E \int_{t_{0}}^{\infty} I(X>u) e^{-\left(u-t_{0}\right)} d u=\bar{F}\left(t_{0}\right) E\left[I\left(X>t_{0}\right)\left(1-e^{-\left(X-t_{0}\right)}\right)\right]
\end{aligned}
$$

and

$$
E \int_{0}^{\infty} f\left(x+t_{0}\right) e^{-x} d x=E\left[e^{-\left(x-t_{0}\right)} I\left(x \geq t_{0}\right)\right]
$$

Then the result is simple to obtain.

$$
\Delta_{F}=0 \text { under } H_{0} \text { and } \Delta_{F}>0 \text { under } H_{1} \text {. To }
$$

estimate $\Delta_{F}$ let $X_{1}, X_{2}, X_{3}, \ldots X_{n}$ be a random sample from

$\mathrm{F}$, then $\mathrm{F}(\mathrm{t})$ will be empirically estimated by $\hat{F}_{n}(t)$ So the empirical form of $\Delta_{F}$ is as follows

$$
\hat{\Delta}_{F}=\frac{1}{n^{2}} \sum_{i, j=1}^{n}\left(\begin{array}{l}
I\left(X_{i}>t_{0}\right) I\left(X_{j}>t_{0}\right) e^{-\left(X_{j}-t_{0}\right)} \\
-\frac{1}{a} K\left(\frac{t_{0}-X_{i}}{a}\right) I\left(X_{j}>t_{0}\right)\left(1-e^{-\left(X_{j}-t_{0}\right)}\right)
\end{array}\right)
$$

here $\hat{f}_{n}\left(t_{0}\right)$ is as follows

$$
\hat{f}_{n}\left(t_{0}\right)=\frac{1}{n a_{n}} \sum_{j=1}^{n} K\left(\frac{t_{0}-X_{j}}{a_{n}}\right)
$$

For more details about $\mathrm{K}$ and the sequence $\left\{a_{n}\right\}$ See [13]. If we choose

$$
\begin{aligned}
& \phi\left(X_{1}, X_{2}\right)=I\left(X_{1}>t_{0}\right) I\left(X_{2}>t_{0}\right) e^{-\left(X_{2}-t_{0}\right)} \\
& -\frac{1}{a_{n}} K\left(\frac{X_{1}-t_{0}}{a_{n}}\right) I\left(X_{2}>t_{0}\right)\left(1-e^{-X_{2}-t_{0}}\right)
\end{aligned}
$$

then $\hat{\Delta}(F)$ equivalents the following U-statistic cf [18].

$$
U_{n}=\frac{1}{\left(\begin{array}{l}
n \\
2
\end{array}\right)} \sum_{R} \psi\left(X_{i}, X_{j}\right) .
$$

Where, the summation over all arrangements of $X_{i 1}, X_{i 2}$, i: $1,2,3, \ldots, \mathrm{n}$. The proof of the following theorem follows from the standard theory of U-statistic, which obtained the asymptotic distribution of $\hat{\Delta}_{F}$.

Theorem As $n \rightarrow \infty$, the random variable $\sqrt{n}\left(\hat{\Delta}_{F}-\Delta_{F}\right)$ is asymptotically normal with mean 0 and variance $\sigma^{2}$ where $\sigma^{2}$ is given in (3.1). Under $H_{0}, \quad \sigma_{0}^{2}$ is given by (3.2).

Proof Using standard U-statistics theory, cf- [18], we need only the asymptotic variance which is equal to

\section{Now since}

$$
\begin{aligned}
& E\left[\phi\left(X_{1}, X_{2}\right) \mid X_{1}\right]=\left[I\left(X_{1} \geq t_{0}\right)+f\left(t_{0}\right)\right] e^{t_{0}} E\left(I\left(X_{2} \geq t_{0}\right) e^{-X_{2}}\right) \\
& -f\left(t_{0}\right) \bar{F}\left(t_{0}\right) \\
& \text { and } \\
& \qquad\left[\phi\left(X_{1}, X_{2}\right) \mid X_{2}\right]=\bar{F}\left(t_{0}\right) I\left(X_{2} \geq t_{0}\right) e^{-\left(X_{2}-t_{0}\right)} \\
& \qquad \mathrm{E}^{-\left(1-e^{-\left(X_{2}-t_{0}\right)}\right) I\left(X_{2} \geq t_{0}\right) f\left(t_{0}\right)}
\end{aligned}
$$

Hence,

$$
\sigma^{2}=V\left\{\begin{array}{l}
\left(I\left(X>t_{0}\right)+f\left(t_{0}\right)\right) E\left(I\left(X>t_{0}\right) e^{-\left(X-t_{0}\right)}\right)+ \\
\bar{F}_{0}\left(t_{0}\right)\left(I\left(X>t_{0}\right)\right) e^{-\left(X-t_{0}\right)}- \\
\left.f\left(t_{0}\right) I\left(X>t_{0}\right)\left(1-e^{-\left(X-t_{0}\right)}\right)-f\left(t_{0}\right) \bar{F}_{0}\left(t_{0}\right)\right)
\end{array}\right\} .
$$

Under $H_{0}$,

$$
\sigma_{0}^{2}=\frac{1}{4} \bar{F}^{4}\left(t_{0}\right)-\frac{1}{12} \bar{F}^{4}\left(t_{0}\right)\left(-6+7 \bar{F}^{-1}\left(t_{0}\right)\right) .
$$

This completes the proof.

It is easy to explain that our proposed test is consistent and unbiased. To perform above test, calculate $\sqrt{\frac{n}{\sigma_{0}^{2}}} \hat{\Delta}_{F}$ and reject $H_{0}$ if this value exceeds $-Z_{\alpha}$, the lower $\alpha$ percentile of the standard normal variate.

The asymptotic Pitman efficiacy of a statistic $T_{n}$ is defined by

$$
\operatorname{eff}\left(T_{n}\right)=\frac{\left|\frac{\partial}{\partial \theta} E\left(T_{n}\right)\right|_{\theta \rightarrow \theta_{0}}}{\sigma_{0}} .
$$

The following families of alternatives are used for efficiacy comparisons :

the Linear failure rate distribution: $\exp \left(-t-\theta t^{2} / 2\right)$, $\mathrm{t} \geq 0, \theta \geq 0$

the Makeham distribution: $e^{-t-\theta\left(t+e^{-t}-1\right)}, \mathrm{t}>0, \theta \geq 0$

the $c^{*}$ distribution

$$
\bar{F}(t)=\left\{\begin{array}{cc}
e^{-t+\theta t^{2}\left(2 t_{0}\right)^{-1}}, & 0 \leq t<t_{0}, 0 \leq \theta \leq 1 \\
e^{-t+\frac{\theta t^{2}}{2} t_{0}}, & t>t_{0}, 0 \leq \theta \leq 1
\end{array}\right.
$$

the null exponential is attained at $\theta=0$.

Carrying out efficacy calculations for the above alternatives , we get

$$
\begin{gathered}
\frac{\sqrt{3} e^{-2 t_{0}}\left(1-2 t_{0}-e^{t_{0}}\left(1-2 t_{0}+2 t_{0}^{2}\right)\right)}{4 \sqrt{e^{-4 t_{0}}\left(-3+7 e^{t_{0}}\right)}}, \\
\frac{e^{-3 t_{0}}}{\sqrt{3} \sqrt{e^{-4 t_{0}}\left(-3+7 e^{t_{0}}\right)}},
\end{gathered}
$$

and 


$$
\begin{aligned}
& \left(\begin{array}{l}
-2+t_{0}+12 t_{0}^{2}-6 t_{0}^{3}-8 t_{0}^{2} e^{t_{0}\left(2+t_{0}\right)}-2\left(-2+t_{0}\right) t_{0}^{2} e^{\frac{1}{2} t_{0}\left(2+t_{0}\right)}+ \\
\left(2-5 t_{0}+10 t_{0}^{2}-4 t_{0}^{3}\right) e^{2 t_{0}^{2}}+\left(2+3 t_{0}-6 t_{0}^{2}+2 t_{0}^{3}\right) e^{3 t_{0}}+ \\
\left(-2-7 t_{0}-12 t_{0}^{2}+8 t_{0}^{3}\right) e^{t_{0}}
\end{array}\right) \times \\
& \left(\frac{4 t_{0}\left(-2+t_{0}\right) \sqrt{\left(-3+7 e^{t_{0}}\right) e^{-4 t_{0}}}}{\sqrt{3} e^{-4 t_{0}}}\right)^{-1}
\end{aligned}
$$

respectively.

For sample sizes $5,6,7 \ldots 50$ and using 5000 replications we tabulate the lower and upper percentiles for $\hat{\Delta}_{F}$ for $t_{0}=0.25$ in table (Appendix). Its powers for sample sizes 10, 20 and 30 for the linear failure rate, weibull and gamma families are given in the following two tables respectively.

\begin{tabular}{|c|c|c|c|c|c|}
\hline \multicolumn{6}{|c|}{ Power estimates of $\hat{\Delta}_{F}\left(t_{0}=\mathbf{0 . 5}\right)$} \\
\hline Distribution & $\theta$ & \multicolumn{4}{|c|}{$\mathrm{n}$} \\
\hline \multirow{4}{*}{ LFR } & \multirow{4}{*}{$\begin{array}{l}2 \\
3 \\
4\end{array}$} & 5 & 10 & 15 & 20 \\
\hline & & 1.000 & 1.000 & 1.000 & 1.000 \\
\hline & & 1.000 & 1.000 & 1.000 & 1.000 \\
\hline & & 1.000 & 1.000 & 1.000 & 1.000 \\
\hline \multirow{3}{*}{ WEIBULL } & 2 & 1.000 & 1.000 & 1.000 & 1.000 \\
\hline & 3 & 1.000 & 1.000 & 1.000 & 1.000 \\
\hline & 4 & 1.000 & 1.000 & 1.000 & 1.000 \\
\hline \multirow{3}{*}{ Gamma } & 2 & 1.000 & 1.000 & 1.000 & 1.000 \\
\hline & 3 & 1.000 & 1.000 & 1.000 & 1.000 \\
\hline & 4 & 1.000 & 1.000 & 1.000 & 1.000 \\
\hline
\end{tabular}

Power estimates of $\hat{\Delta}_{F}\left(t_{0}=\mathbf{0 . 2 5}\right)$
\begin{tabular}{|l|l|l|l|l|l|}
\hline Distribution & $\theta$ & \multicolumn{4}{|c|}{$\mathrm{n}$} \\
\hline \multirow{4}{*}{ LFR } & & 5 & 10 & 15 & 20 \\
\cline { 2 - 6 } & 2 & 1.000 & 1.000 & 1.000 & 1.000 \\
\cline { 2 - 6 } & 3 & 1.000 & 1.000 & 1.000 & 1.000 \\
\cline { 2 - 6 } & 4 & 1.000 & 1.000 & 1.000 & 1.000 \\
\hline \multirow{3}{*}{ Weibull } & 2 & 1.000 & 1.000 & 1.000 & 1.000 \\
\cline { 2 - 6 } & 3 & 1.000 & 1.000 & 1.000 & 1.000 \\
\cline { 2 - 6 } & 4 & 1.000 & 1.000 & 1.000 & 1.000 \\
\hline \multirow{3}{*}{ Gamma } & 2 & 1.000 & 1.000 & 1.000 & 1.000 \\
\cline { 2 - 6 } & 3 & 1.000 & 1.000 & 1.000 & 1.000 \\
\cline { 2 - 6 } & 4 & 1.000 & 1.000 & 1.000 & 1.000 \\
\hline
\end{tabular}

Thus we have shown that, based on Monte Carlo methods, our test statistic has a full powers for all alternatives.

Example 3.3 The data of this example consist of the survival times of 40 patients suffering from blood cancer (Leukemia) from one of the hospitals in Saudi Arabia, [14]. The orderd life times (in days) are:

$\begin{array}{lllllllll}115.0 & 181.0 & 255.0 & 418.0 & 441.0 & 461.0 & 516.0 & 739.0 & 743.0\end{array}$ 789.0807 .0865 .0924 .0983 .01024 .01062 .01063 .01165 .0

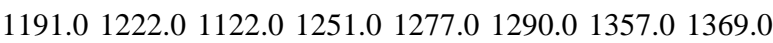
$\begin{array}{llllllll}1408.0 & 1455.0 & 1478.0 & 1549.0 & 1578.0 & 1578.0 & 1599.0 & 1603.0\end{array}$ 1605.01696 .01735 .01799 .01815 .01852 .0

To apply the test we choose $t_{0}=1.137$ ( $\approx 3$ years). We obtain the value of our test for the blood cancer data with a corresponding one side and 0.05 level of significant hence
$H_{0}$ is not rejected, agreeing with the conclusion of [14] and [3]

\section{REFERENCES}

[1] Abouammoh, A. M. and Ahmed, A. N. (1988). The new better than used failure rate class of life distribution. Adv. Prob., 20, 237-240.

[2] Ahmad, I.A. (1994). A class of statistics useful in testing increasing failure rate average and new better than life distributions, J. Statist. Planning Inf. 41 141149.

[3] Ahmad, I.A. (2001). Moment inequalities of aging families of distributions with hypotheses testing applications, J. Statist. Planning Inf. $92121-132$.

[4] Ahmad, I. A. (1998). Testing whether a survival distribution Is new better than used of an unknown specified age. Biometrika. 85, 451-7.

[5] Ahmed. I. A., Ibrahim. A. A. and Mugdadi. A. R. (2001). A goodnees if fit approach to major life testing problems. International J. of reliability and applications, $2,2,81-97$

[6] Ahmed H.El-Bassiouny, Ammar M.Sarhan, M.AlGarian (2004). Testing exponentiality against NBUFR (NWUFR), Appl. Math. and Computat., 149, 2, 351358.

[7] Barlow, R. E. and Proschan, F. (1981). Statistical Theory of Reliability and Life Testing. To Begin with. Silver Spring, M D.

[8] Bryson, M.C. and Siddiqui, M.M. (1969). Some criteria for aging. J. Amer. Statist. Asoc., 64, 1472-1483.

[9] Cao, J. and Wang, Y. (1991). The NBUC and NWUC classes of life distributions. J. Appl. Prob., 28, 473-479.

[10] Deshpande, J.V., Kochar, S.C. and Singh, H. (1986). Aspects of positive aging. J. Appl. Prob., 23, 748-758.

[11] Ebrahimi, N. and Habbibullah, M. (1990). Testing whether the survival distribution is new better than used of specified age, Biometrika 77, 212-215.

[12] Elbatal, I (2007). Some Aging Classes of Life Distributions at Specific Age. International Mathematical Forum, 2, 29, 1445-1456.

[13] Hardle,W. Smoothing Techniques With Implementation In

S. Spring-Verlag (1991), New York.

[14] Hendi, M. and Abouammoh, A. (2001). Testing new better than renewal used life distributions based on $\mathrm{U}$ test. Comm. Statist. Theory. Math 30, 10 2135-2148.

[15] Hollander, M. Proschan, F. (1972). Testing whether new is better than used, Ann. Math. Statist. 43, 1136-1146.

[16] Hollander, M. Park, D. and Proschan, F. (1986). A class of life distributions for aging. J. Am. Statist. Ass. 81, 91-95.

[17] Ibrahim A. Alwasel and Ahmed H. El-bassiouny. (2001) Testing whether a survival function is new better than used of a specified age, ISSR. Cairo Univ., 45, 2, 155-162. 
[18] Lee, A. J. U-Statistics, Marcel Dekker (1989), New York, NY.

[19] Li Zehui and Li Xiaohu (1998). $\left\{\mathrm{IFR}^{*} t_{0}\right\}$ and $\left\{\mathrm{NBU}^{*}\right.$ $\left.t_{0}\right\}$ classes of life distributions. J. Statist. Planning Inf. 70, 191-200.

[20] Mahmoud, M. A. W. and Abdul Alim, N. A (2002) On testing exponentiality against nbarfr life distributions. STATISTICA, anno LXII, n, 4, pp 599-610.

[21] Mahmoud, M.A.W. Abdul Alim, N.A. and Diab L.S. (2009): On The New Better Than Used Renewal Failure Rate at Specied Time. EQC 24, 1, 87-99.

\section{Appendix}

Table Percentiles for $\hat{\Delta}_{F}\left(t_{0}=\mathbf{0 . 2 5}\right)$

\begin{tabular}{|c|c|c|c|c|c|c|c|}
\hline & & 5 & .10 & .90 & .95 & .98 & \\
\hline 5 & 7727 & 295 & 007 & 410 & $100 \mathrm{~s}$ & 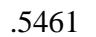 & $\sigma e$ \\
\hline 6 & -.0670 & -.0179 & & & & & \\
\hline 7 & & & & & & & \\
\hline 8 & & & & & & & \\
\hline 9 & & & & & & & \\
\hline 10 & & & & & & & 60 \\
\hline 11 & & & & & & & \\
\hline 12 & & & & & & & .4 \\
\hline 13 & -.03 & .0133 & & .29 & & & .417 \\
\hline 14 & -.02 & .01 & & 2920 & & .3 & .404 \\
\hline 15 & -.02 & .0227 & & 28 & & & .403 \\
\hline 16 & -.01 & .0248 & & .28 & & & .387 \\
\hline 17 & -.01 & .0231 & & & & & .372 \\
\hline 18 & -.01 & & & & & & \\
\hline 19 & -.01 & & & & & & \\
\hline 20 & -.00 & & & & & & \\
\hline 21 & -.0086 & .0290 & & & & & 90 \\
\hline 22 & -.0066 & & & & & & \\
\hline 23 & -.0028 & & & & & & \\
\hline 4 & -.0086 & & & & & & 27. \\
\hline & -.00 & & & & & & \\
\hline & -.00 & & & & & & \\
\hline & & & & & & & \\
\hline & .00 & & & & & & \\
\hline 2 & .00 & & & & & & \\
\hline & -.00 & & & & & & \\
\hline & & & & & & & \\
\hline & .00 & & & & & & \\
\hline & .00 & & & & & & \\
\hline & & & & & & & \\
\hline & & & & & & & \\
\hline & & & & & & & \\
\hline & & & & & & & \\
\hline & & & & & & & \\
\hline & & & & & & & \\
\hline & & & & & & & \\
\hline & & & & & & & \\
\hline & & & & & & & \\
\hline 43 & & & & & & & $x$ \\
\hline 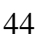 & & & & & & & 02 \\
\hline 15 & & & & & & & .2720 \\
\hline 7 & & & & & & & .2676 \\
\hline 4 & & & & & & & .2670 \\
\hline 48 & .02 & & & & & & \\
\hline 49 & .02 & & & & & & 2600 \\
\hline 50 & .0202 & .0506 & .0675 & 6 & 226 & 423 & .2565 \\
\hline
\end{tabular}

[22] Parameshwar V. Pandit (2004). A note on testing exponentiality against new better than used of specified age, Econ. Quality Control, 19, 2, 205-213.

[23] Proschan, F. Pyke, R. (1967). Tests for monotone failure rate, Fifth Berkely Sympo.3 293 -313.

[24] Rolski, T. (1975). Mean residual life. Bulletin of the International Statistical Institute. 46,266-270. 